\title{
Historia preliminar de incendios y prácticas (multi) culturales en la cuenca media del río Cachapoal $\left(34^{\circ} \mathrm{S}\right)$, Chile central
}

\author{
Preliminary fire history and (multi) cultural practices in the Cachapoal river's middle basin \\ $\left(34^{\circ} \mathrm{S}\right)$, Central Chile
}

\author{
Carlos Bustos-Schindlera, b*, Carlos Le Quesne ${ }^{\mathrm{a}}$, Mauro E González ${ }^{\mathrm{a}, \mathrm{c}}$, M Eugenia Solarid \\ *Autor de correspondencia: aUniversidad Austral de Chile, Facultad de Ciencias Forestales, casilla 567, Valdivia, Chile, \\ tel.-fax: 56-63-221228, carlosbustos@bosquenativo.cl \\ bUniversidad Austral de Chile, Centro Transdisciplinario de Estudios Ambientales (CEAM), Valdivia, Chile. \\ 'Núcleo Científico Milenio FORECOS, Valdivia, Chile. \\ ${ }^{d}$ Universidad Austral de Chile, Facultad de Filosofía y Humanidades, Valdivia, Chile.
}

\begin{abstract}
SUMMARY
This work was developed in the Cachapoal river basin, Andean Mountain, of Central Chile. In this basin the relations between anthropogenic factors and fire frequency in Austrocedrus chilensis forests were explored. Fire history of this area was reconstructed using cypress cross-sections dating, historical revision and local people interviews. Archaeological information reports human occupation of this zone since the Early Holocene. Starting in the XVII century, this area was used by Jesuits mainly for cattle ranching activities. Later, the property was passed to private owners who continued using this area for agricultural and cattle purposes. The tree-ring chronology presents a length of 754 years (1249 -2003). The oldest fire dated occurred in 1389 and the latest fire in 1923. Based on the numbers of dated samples, the main fires during this period occurred in 1484, 1526, 1575, 1800 and 1893 . The fire in this region is an important process in the ecosystem functioning and a major landscape modeler. The interviews showed that fire had been an element widely used by rural population for heating and cooking functions, location (smoke signals) and animal hunting. These evidences allow making a close connection between anthropogenic activities and climate in the occurrence and frequency of fires.
\end{abstract}

Key words: Austrocedrus chilensis, anthropogenic influence, Cachapoal basin, Central Chile.

\section{RESUMEN}

Este trabajo se desarrolló en la porción media de la cuenca del río Cachapoal, cordillera andina de Chile central ( $\left.34^{\circ} \mathrm{S}\right)$. En esta zona se exploraron las relaciones entre factores antropogénicos y frecuencia de incendios en bosques de Austrocedrus chilensis (ciprés de la cordillera). Se reconstruyó una cronología preliminar de incendios de esta cuenca mediante el fechado de cicatrices de fuego en rodelas de ciprés, revisión histórica y entrevistas a habitantes de la zona. La revisión historiográfica informa de la ocupación humana de esta zona desde el Holoceno temprano. A partir del siglo XVII, el área fue utilizada por la orden jesuita con fines principalmente ganaderos y mineros, para luego ser traspasada a terratenientes y propietarios privados que siguieron utilizando el área extensivamente en actividades agrícola-ganaderas. La cronología de ancho de anillos presentó una longitud de 754 años (1249-2003). El incendio más antiguo fue datado en 1389, y el más reciente en 1923. Los principales incendios durante este periodo ocurrieron en 1484, 1526, 1575, 1800 y 1893. El fuego en esta zona es un proceso importante en el funcionamiento del ecosistema y un claro modelador del paisaje. La información recopilada mediante entrevistas indica que el fuego ha sido un elemento ampliamente usado por la población rural, especialmente para fines de calefacción y preparación de alimentos, ubicación (señales de humo) y despeje para la búsqueda de animales. Los resultados sugieren una estrecha relación entre las actividades antropogénicas y la variabilidad climática, con la ocurrencia y frecuencia de incendios, y muestran el potencial que posee el registro de anillos para reconstruir la historia natural y cultural de Chile central.

Palabras clave: Austrocedrus chilensis, influencia antropogénica, cuenca del Cachapoal, Chile central.

\section{INTRODUCCIÓN}

El análisis de documentación histórica y el uso de técnicas dendroecológicas para lograr la datación de cicatrices de fuego (Veblen et al. 2004) permiten desarrollar historias de incendios, como la que se expone a continuación.
La dendrocronología se ocupa del fechado de eventos ambientales y variaciones climáticas a través de anillos de crecimiento anual de los árboles (Fritts 1976). Dentro de dicha disciplina se encuentra la dendropirocronología (Grissino-Mayer 2006), la cual se ocupa de datar y estudiar cambios pasados y presentes en los regímenes de 
incendios (Grissino-Mayer et al. 2001). En incendios de baja a moderada intensidad los árboles pueden producir marcas o cicatrices en el fuste (Lachmund 1921, Guyette y Dey 1995), las cuales son causadas cuando parte del cambium se muere o quema. Johnson y Van Wagner (1978) mencionan que el estudio de regímenes de incendio no es sólo el análisis de las cicatrices en las muestras dendrocronológicas, sino también el análisis de cualquier evidencia de variaciones en el crecimiento de los anillos que registre la ocurrencia de ellos.

En Chile central el fuego es un proceso común, tanto como en otras regiones de clima mediterráneo (Montenegro et al. 2002). Un factor de gran importancia en el aumento de la frecuencia de incendios en estas regiones, es el impacto antropogénico al despejar vegetación nativa para el desarrollo de agricultura. En el paisaje mediterráneo de Chile central se han reemplazado vastas extensiones de vegetación leñosa silvestre por parches de vegetación dispersa en un mosaico entremezclado con terrenos agrícolas, conjuntos de maleza y vegetación suburbana con menor índice de combustión, al igual que desarrollos suburbanos no inflamables. Por ello, puede esperarse que en áreas cercanas a centros urbanos exista un bajo intervalo de retorno del fuego (Aravena $e t$ al. 2003, Montenegro et al. 2007).

Los bosques de Austrocedrus chilensis (D. Don) Pic. Ser. et Bizz. (ciprés de la cordillera) pertenecen a los ecosistemas mediterráneos afectados por incendios. Esta especie presenta la distribución más septentrional de las coníferas del Cono Sur de Sudamérica (figura 1), ocupando preferentemente ambientes andinos en sustrato rocoso, por lo que es considerada una especie rústica adaptada para crecer en ambientes xéricos (Le Quesne 1988). Austrocedrus chilensis presenta anillos anuales definidos y se ha empleado en la reconstrucción de historias de incendios, principalmente en la parte norte de la Patagonia argentina (Kitzberger et al. 1997, Veblen et al. 1999, Veblen y Kitzberger 2002) y recientemente en Chile central por Aravena et al. (2003).

Este trabajo inicial combina el análisis de las cicatrices de incendio en árboles de Austrocedrus chilensis con una revisión histórica y entrevistas semiestructuradas a habitantes locales para reconstruir la historia de incendios de esta zona geográfica. Así, el objetivo general es explorar preliminarmente las relaciones de los factores antropogénicos con la frecuencia de incendios en bosques de Austrocedrus chilensis en cuatro localidades de la cuenca media del río Cachapoal.

\section{MÉTODOS}

Área de estudio. Esta investigación se desarrolló en la cuenca media del río Cachapoal (CCH), donde se analizaron cuatro sitios, en altitudes que oscilan desde los 1.400 a los 1.800 m s.n.m. (cuadro 1). Los sitios se ubican en la cordillera andina de Chile central $\left(34^{\circ} \mathrm{S}\right)$, a $50 \mathrm{~km}$ al este de la ciudad de Rancagua (figura 1).

El clima en el área de estudio corresponde a templado de montaña con cuatro meses secos, caluroso en verano, con un invierno lluvioso y precipitaciones en forma de nieve. La precipitación media anual va desde los $800 \mathrm{~mm}$ en las partes bajas hasta los $1.500 \mathrm{~mm}$ en las más altas. Las temperaturas máximas promedio de verano en las zonas de

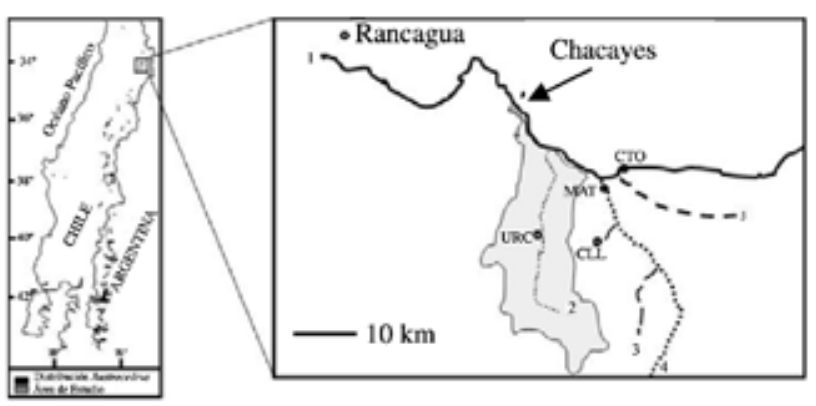

Figura 1. Distribución de Austrocedrus chilensis y área de estudio en la cuenca media del río Cachapoal. Se muestran los sitios de estudio (Urriola (URC), Matancilla (MAT), Cipresito (CTO) y Cipresillo (CLL)), y los ríos que conforman la cuenca (1: Cachapoal, 2: Cipreses, 3: Blanco, 4: Cortaderal y 5: Las Leñas). Con la flecha negra se muestra la localidad de Chacayes y en gris claro la Reserva Nacional Río de Los Cipreses (RNRC).

Austrocedrus chilensis distribution in Chile and Argentina, and the Cachapoal middle basin study area. Study sites (Urriola, Matancilla, Cipresito and Cipresillo) and the rivers that drain the zone (1: Cachapoal, 2: Cipreses, 3: Blanco, 4: Cortaderal and 5: Las Leñas) are indicated. The black arrow shows the location of Chacayes. The gray area is the basin surface occupied by the Reserva Nacional Río de Los Cipreses (RNRC).

Cuadro 1. Características de los sitios muestreados en la cuenca media del Cachapoal.

Characteristics of the sampling sites in the Cachapoal middle basin.

\begin{tabular}{|c|c|c|c|c|c|c|}
\hline Sitio & Código & $\mathrm{N}^{\circ}$ Rodelas & $\begin{array}{c}\text { Latitud } \\
(\mathrm{S})\end{array}$ & $\begin{array}{c}\text { Longitud } \\
\text { (O) }\end{array}$ & $\begin{array}{c}\text { Altitud } \\
\text { (m s.n.m.) }\end{array}$ & Ambiente $^{\mathrm{a}}$ \\
\hline Urriola & URC & 9 & $34^{\circ} 27^{\prime}$ & $70^{\circ} 25^{\prime}$ & $1.600-1.700$ & Pendiente escarpada \\
\hline Matancilla & MAT & 7 & $34^{\circ} 20^{\prime}$ & $70^{\circ} 19^{\prime}$ & $1.400-1.600$ & Talud rocoso \\
\hline Cipresito & CTO & 10 & $34^{\circ} 21^{\prime}$ & $70^{\circ} 18^{\prime}$ & $1.400-1.550$ & Pendiente escarpada \\
\hline Cipresillo & CLL & 1 & $34^{\circ} 28^{\prime}$ & $70^{\circ} 19^{\prime}$ & $1.600-1.800$ & Talud rocoso \\
\hline
\end{tabular}

${ }^{a} \mathrm{El}$ ambiente se refiere a la disposición de la forma de terreno en el cual se sitúan los bosques de Austrocedrus chilensis estudiados. 
menor altitud oscilan entre los 19 y $21^{\circ} \mathrm{C}$ y las mínimas tienen un promedio de 5 a $7^{\circ} \mathrm{C}$ (Novoa y Villaseca 1989). La cuenca del río Cachapoal tiene una superficie de 6.370 $\mathrm{km}^{2}$. Nace de las cumbres glaciales Pico del Barroso y Nevado de los Piuquenes, y recibe las aguas de los ríos Las Leñas, Cortaderal, Los Cipreses y Coya (Niemeyer y Cereceda 1984) (figura 1). Aravena et al. (2003) mencionan la caída de rayos como fuentes posibles de ignición natural en esta zona de Chile central.

Es característico de esta zona, a los 1.000 m s.n.m., un bosque esclerófilo secundario compuesto entre otras especies por: Quillaja saponaria Mol. (quillay), Lithraea caustica Hook. et Arn. (litre), Aristotelia chilensis (Mol.) Stuntz (maqui), Kageneckia oblonga R. et P. (bollén), Retanilla ephedra (Vent.) Brongn. (retamilla). A medida que se incrementa la altitud, hacia los 1.400 m s.n.m., desaparece el bosque de quillay y litre, para dar paso a formaciones abiertas de Kageneckia angustifolia D. Don. (frangel u olivillo de la cordillera) (Barría 2007). A partir de $1.500 \mathrm{~m}$ s.n.m. crecen las primeras agrupaciones de Austrocedrus chilensis cercanas al curso del río y dispersas en bosquetes sobre laderas donde la especie adopta un hábito achaparrado hacia el límite altitudinal arbóreo, estimado en 2.200 m s.n.m. en esta zona. Especies como Maytenus boaria Mol. (maitén), Guindilia trinervis Gillies ex Hook. et Arn. (guindillo), Colliguaja salicifolia Gillies et Hook (colliguay macho), Schinus montanus (Phil.) Engler (litrecillo) y Azara petiolaris (D. Don.) Johnst. (lilén), presentes también en el bosque esclerófilo, cohabitan en los bosques abiertos de Austrocedrus chilensis. Cercanía a manantiales o mallines se encuentran pequeños grupos de Escallonia revoluta (R. et P.) Pers. (lun o corontillo) asociados a hierbas acuáticas. En extensiones planas de la caja fluvial se presenta también Cortaderia araucana Stapf. (cola de zorro). Finalmente, es propio de los pisos altitudinales superiores la vegetación de pradera altoandina (Le Quesne et al. 1999).

Historia de la influencia antropogénica en la cuenca. Para la recopilación de información etnográfica se utilizó la entrevista semiestructurada, método de investigación cualitativo en donde se realizan reiterados encuentros cara a cara entre el investigador y los informantes, los cuales están dirigidos a la comprensión de las perspectivas que tienen estos últimos en relación a sus vidas, experiencias o situaciones, expresadas con sus propias palabras (Taylor y Bogdan 1987). Desde esa óptica metodológica, se entrevistaron a seis personas de Chacayes, comunidad aledaña a la Reserva Nacional Río de Los Cipreses (RNRC) que han trabajado en las inmediaciones de las poblaciones de Austrocedrus chilensis de la cuenca media del río Cachapoal o en la otrora hacienda Chacayes (cuadro 2). Estas entrevistas incluyeron los siguientes temas: i) vida en la hacienda Chacayes durante el siglo XX, ii) actividades que se realizaban en ella, iii) actividades que se realizaban hacia los cajones en esos años, iv) conocimiento de ocurrencia de incendios y sus causas. Las respuestas fueron organizadas en tres categorías: historia de la hacienda, usos del fuego y causas de incendios.

La revisión de la documentación histórica referente a la economía, uso de la tierra y fotografías de la época (fines del siglo XIX e inicios del XX) sirvió para compilar y sistematizar la información de los asentamientos históricos en esta cuenca. La investigación comprendió antiguos títulos de dominio de las haciendas aledañas a la cuenca, documentos generados por naturalistas e investigadores que caracterizaron la región desde la mitad del siglo XIX y bibliografía que poseía información acerca de la actividad económica de la época. Esta revisión se realizó principalmente en los archivos de la Biblioteca Nacional, Sección Chilena, y Sala José Toribio Medina. Las fotografías fueron obtenidas de la colección de la familia Bustamante, ex dueños de la hacienda Chacayes, la que facilitó estos documentos al administrador de la RNRC. Finalmente se revisaron textos de los jesuitas en la Biblioteca de la Universidad Alberto Hurtado, en Santiago. Este trabajo bibliográfico se basó fundamentalmente en el documento técnico desarrollado por Arrué (2000).

Cronología de incendios. Se analizaron 35 secciones transversales de Austrocedrus chilensis, con evidencias de incendio (figura 2), provenientes de árboles muertos que se presentan dispersos en el paisaje. Estas muestras se obtuvieron durante las campañas de terreno realizadas por Le Quesne et al. (2006) en la cuenca del río Cachapoal, que comprendieron las localidades indicadas en el cuadro 1.

Cuadro 2. Características de los entrevistados en la localidad de Chacayes.

Personal information of persons interviewed in the town of Chacayes.

\begin{tabular}{lccl}
\hline Nombre & $\begin{array}{c}\text { Edad } \\
(\text { años })\end{array}$ & $\begin{array}{c}\text { Grupo familiar } \\
\left(\mathrm{N}^{\circ} \text { personas }\right)\end{array}$ & Actividad \\
\hline Joaquín López & 42 & 6 & Manutención RNRC, hijo de arriero de la hacienda Chacayes \\
Salomé Silva & 37 & 5 & Dueña de casa, hija de administrador de la hacienda Chacayes \\
Ángel Lara & 58 & 5 & Arriero, realiza viajes ecuestres a Cajón Cipreses \\
Ramón López & 85 & 8 & Arriero jubilado de la hacienda Chacayes \\
Rosa Miranda & 76 & 8 & Dueña de casa, ex trabajadora de la hacienda Chacayes \\
Domitila Sánchez & 82 & 7 & Dueña de casa, ex trabajadora de la hacienda Chacayes \\
\hline
\end{tabular}


Para la observación detallada de los anillos anuales las muestras fueron pulidas con lijas de granulometría progresivamente menor. El lijado, así como el fechado visual, se llevó a cabo utilizando las técnicas descritas por Stokes y Smiley (1968). El programa computacional COFECHA (Holmes et al. 1986) fue usado para asegurar la exactitud del fechado relativo y absoluto entre las muestras. Tomando en cuenta que esta cronología fue desarrollada a partir de árboles muertos, para fechar cada muestra se utilizó la cronología maestra de Urriola, desarrollada por Le Quesne et al. (2006).

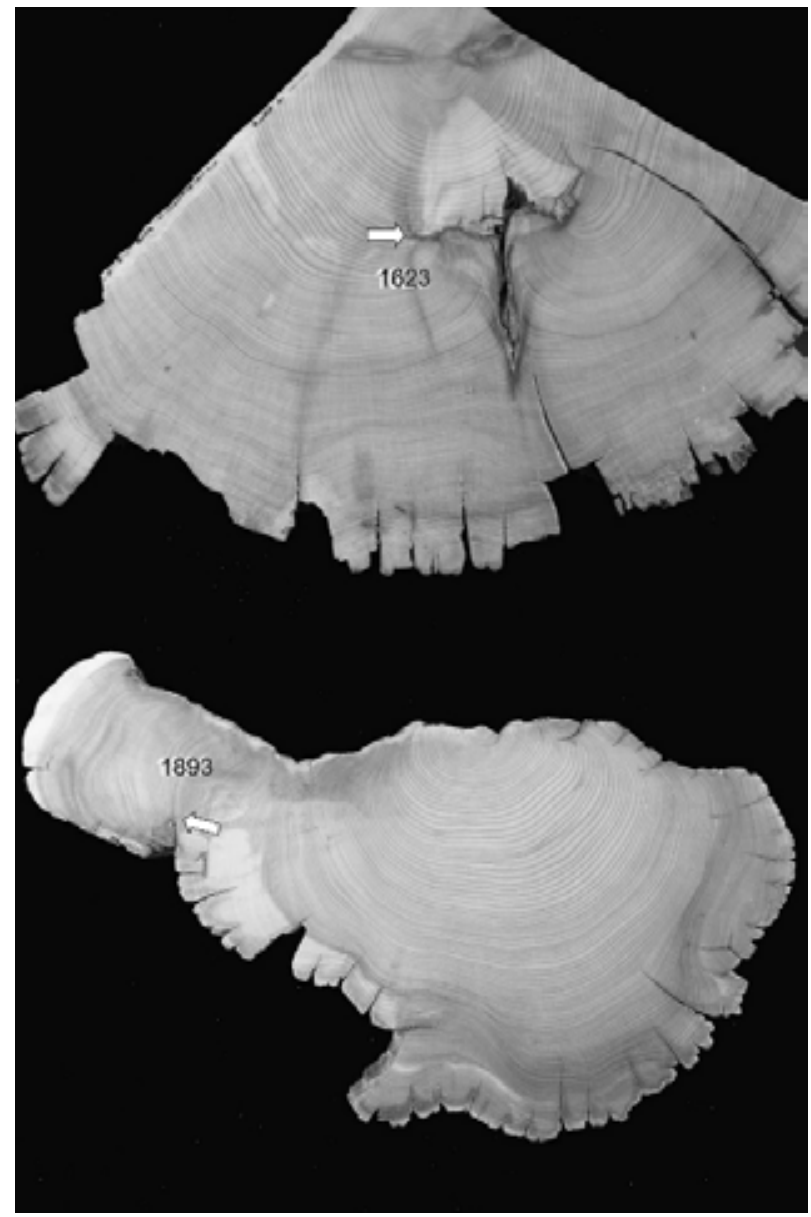

Figura 2. Rodelas de Austrocedrus chilensis que presentan cicatrices de incendio en la cuenca media del Cachapoal.

Austrocedrus chilensis samples of the middle Cachapoal basin presenting fire scars.

\section{RESULTADOS}

Influencia antropogénica. Desde hace más de 9.000 años Chile central ha sido testigo de la presencia humana (Fallabella 1994, Núñez et al. 1994, Aravena et al. 2003). Los cazadores recolectores del Holoceno temprano (Arcaico temprano), probablemente hayan conocido el lugar cuando el derretimiento de los hielos permitió que grandes extensiones de terreno pudieran ser ocupadas por el ser humano (Falabella 1994). A partir del Holoceno medio, los grupos humanos que frecuentaron la cordillera generaron sistemas de caza y recolección complementarios, generando los primeros contactos trasandinos por diferentes lugares de esta cuenca, entre los cuales pudo haberse empleado el conocido paso de Las Leñas (Falabella 1994, Arrué 2000). Tanto los primeros grupos de cazadores recolectores, como los que posteriormente pertenecían a una estrategia agropastoril, ocuparon los diversos ambientes de la cuenca, asentándose probablemente en verano en sectores cordilleranos y en invierno en los valles bajos, practicando así una trashumancia estacional. Estos grupos pertenecieron progresivamente tanto a las culturas Bato, Aconcagua y Guaquivilo. Esta trashumancia los llevó a los cajones cordilleranos para la caza y la recolección de frutos (Arrué 2000). Para el período histórico, algunos autores, basados en archivos de la época, sitúan en estos cajones cordilleranos a un grupo denominado Chiquillanes, que practicaban la extracción pampeana (se caracterizaban por ser cazadores de guanacos, huemules, ñandúes, pumas y aves, además de ser recolectores de especias) (Latcham 1928) y manejaban el fuego de una forma prolija (Medina 1952). Por lo tanto, sería posible atribuir a estas prácticas culturales de manejo de fuego las causas de los eventos de incendio anteriores al año 1600.

A partir de 1604, esta área fue utilizada por la orden Jesuita con fines principalmente ganaderos y una incipiente minería hasta 1665 (Fuenzalida 1919). Los jesuitas tuvieron que adquirir éstas y otras propiedades agrícolas para hacer frente a los gastos que demandaron la fundación de los colegios de San Miguel y de Concepción. Pronto tendrían en sus diferentes haciendas ganado mayor y menor, molinos, curtiembres, viñas, bodegas, herrerías, entre otros adelantos. Para habilitar estos terrenos y desarrollar estas tecnologías, esta orden religiosa usó el fuego para despejar terreno (Hanisch, 1974). En 1665 los jesuitas venden los terrenos de la Hacienda Chacayes a Bartolomé Maldonado de Madrigal y Suárez, quien en diciembre de ese mismo año compra las estancias de Cauquenes, Apaltas y Requínoa. Desde este momento se puede considerar la sección de Los Chacayes como parte de la gran hacienda de Cauquenes. En esos años Bartolomé Maldonado de Madrigal era Secretario de la Real Audiencia por título real que su padre obtuvo para sí y sus descendientes. Desde fines del siglo XVIII esta zona de la cuenca se subdivide en dos grandes haciendas (Chacayes y Cauquenes) y es traspasada a propietarios privados que prosiguieron utilizando el área extensivamente en actividades agrícola-ganaderas (Echevarría 1988, Falabella 1994, Arrué 2000). La intervención antrópica en la zona se manifiesta a través de tocones, fragmentos de madera seca con rastros de carbón y cicatrices en la corteza de árboles vivos (Le Quesne et al. 1999, 2000). Estas manifestaciones se presentan en la parte oriental del cajón Cipreses los años 1716 y 1845, mientras que Barría 
(2007) menciona las mismas evidencias en 1800, pero en la parte poniente de este cajón, específicamente en el rodal adyacente a los antiguos corrales de Urriola (URC). Estos corrales fueron usados por los arrieros para juntar al ganado en épocas estivales a partir de fines del siglo XVI hasta fines del siglo XX (cuadro 3, figura 3). Las diferentes familias que fueron dueñas de este territorio concentraron sus recursos en la cría y venta de ganado, en especial del bovino. Para ello comenzaron a intervenir fuertemente el medio ambiente circundante, en especial los valles que se emplazan en los cajones que conforman la cuenca del Cachapoal (Yrarrázabal 1940, Arrué 2000).

Las entrevistas realizadas, que abarcan aproximadamente los últimos 80 años de asentamiento en la zona, atestiguaron la combustión de vegetación leñosa para fogatas (calefacción y cocina), ubicación de arrieros, despeje y búsqueda de animales. Según los entrevistados, estos incendios fueron originados en forma natural y antrópica, y estos últimos a su vez pudieron ser accidentales o intencionales. No se logró obtener con precisión la fecha de los incendios naturales, mas lo relevante es la confirmación por parte de uno de los entrevistados de la presencia de este tipo de fenómenos en la zona. El cuadro 4 resume cómo las respuestas de los entrevistados fueron clasificadas para obtener la información necesaria para conocer sobre los orígenes y usos del fuego en la cuenca.

Las remodelaciones y construcciones de casas patronales marcan el siglo XX. El uso de Austrocedrus chilensis para estos fines es ampliamente documentado. El traslado de esta noble madera se realizó por vía fluvial (Arrué 2000). Don Andrés Bustamante fue el último dueño de la hacienda Chacayes (1962-1967), quien concentró su producción ganadera en ovinos, reemplazando al ganado bovino por 8.000 lanares. Luego de la expropiación de los terrenos de la cuenca por parte de la Corporación de Reforma Agraria (CORA) en 1967, en esta zona se

Cuadro 3. Hitos históricos de la cuenca del río Cachapoal.

Historical milestones of Cachapoal basin.

\begin{tabular}{|c|c|c|}
\hline Año & Hito & Autor(es), año \\
\hline 9.000 a.C. & Habitantes del Holoceno temprano & Núñez et al. 1994 \\
\hline 90 a.C. a 900 & Culturas Bato & Falabella y Stehberg 2000 \\
\hline $900-1100$ & Cultura Aconcagua & Durán y Planella 2000 \\
\hline $1100-1600$ & Cultura Guaiquivilo & Falabella 1994 \\
\hline 1604 & Llegada de Jesuitas & Hanisch 1974 \\
\hline 1665 & Venta de la propiedad jesuita a B. Maldonado & Fuenzalida 1919 \\
\hline 1736 & Comienza mayorazgo de familia Larraín & Yrarrázabal 1940 \\
\hline 1769 & Intento indígena de internarse en la cuenca & Arrué 2000 \\
\hline 1778 & Segundo intento de internación indígena & Arrué 2000 \\
\hline 1820 & Crianza de 10.000 bovinos en hacienda Chacayes & Falabella 1994 \\
\hline 1830 & Abolición del mayorazgo & Larraín de Castro 1982 \\
\hline 1831 & Visita de C. Gay & Gay 1980 \\
\hline 1845 & Comienzo aumento del precio de las carnes & Arrué 2000 \\
\hline 1841 & Visita de I. Domeyko & Domeyko 1849 \\
\hline 1856 & Mejora de los Baños de Cauquenes & Echevarría 1988 \\
\hline 1858 & Visita de J.A. Pissis & Pissis 1860 \\
\hline 1867 & Venta de la hacienda de Chacayes a Apolinario Soto & Arrué 2000 \\
\hline 1875 & Visita de R.A. Philippi & Philippi 1875 \\
\hline 1876 & Máximo precio de las carnes & Yrarrázabal 1940 \\
\hline 1880 & A. Soto cede mulares y caballares para el ejército & Echevarría 1988 \\
\hline 1893 & Reemplazo de ganadería por agricultura mixta & Falabella 1994 \\
\hline 1902 & Construcción casa patronal, transporte fluvial de cipreses & Arrué 2000 \\
\hline 1910 & Construcción de acueducto & Echevarría 1988 \\
\hline 1921 & Llegada de O. France, dedicándose a la ganadería & Arrué 2000 \\
\hline 1928 & Descripción de indígenas cordilleranos, los Chiquillanes & Latcham 1928 \\
\hline 1943 & Remodelación de casa patronal por C. Azócar & Arrué 2000 \\
\hline 1953 & Jorge Labatú posee 3.000 cabezas de ganado & Echevarría 1988 \\
\hline 1962 & A. Bustamente cría 1.000 bovinos y 8.000 ovejas & Arrué 2000 \\
\hline 1967 & CORA expropia el fundo Chacayes & Ángel Lara, com. pers. \\
\hline 1983 & Creación de la Reserva Nacional Río de Los Cipreses & Arrué 2000 \\
\hline
\end{tabular}




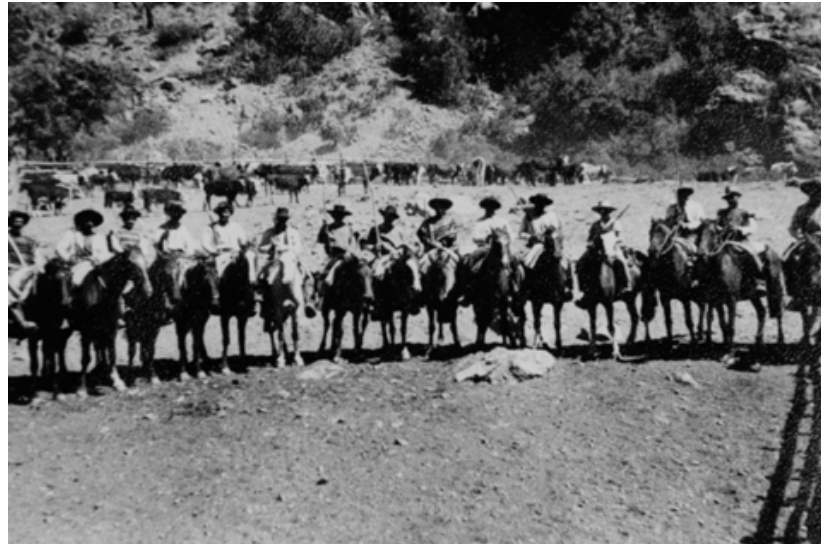

Figura 3. Fotografía de arrieros a fines del siglo XIX en los corrales de Urriola. Gentileza de Julio César Vergara Zapata, ex administrador de la Reserva Nacional Río de Los Cipreses.

Photographs of muleteers, late nineteenth century in Urriola corrals. Courtesy of Julio César Vegara Zapata, former Administrator of the Reserva Nacional Río de Los Cipreses.

emplazó la RNRC, con una superficie de 36.882,5 ha. Esta última a su vez se incorporó en 1983 al Sistema Nacional de Áreas Silvestres Protegidas del Estado (SNASPE), con la finalidad de conservar la flora, fauna e historia de la cuenca andina de río Cachapoal.

Reconstrucción dendrocronológica preliminar de la historia de incendios. La cronología de ancho de anillos de Austrocedrus chilensis se extiende desde 1249 hasta 2003 y gran parte de las muestras recolectadas que presentaron cicatrices de fuego (27/35) fueron datadas satisfactoriamente, así como la mayoría de éstas sobrepasaron los 100 años de longitud, a excepción de la muestra URC322 (79 años). La longitud media de las muestras fue de 215 años. Dentro de ellas hubo ocho muestras que evidenciaron dos eventos de incendio, dos rodelas con tres eventos y las restantes con sólo una marca de fuego. Los años de incendio presentes en más de una muestra fueron 1484, 1526, 1575, 1800 y 1893 (figura 4). Esta última fecha fue la que estuvo representada con el mayor número de muestras, con cinco diferentes rodelas que la contenían en dos sitios distintos y contiguos (MAT y CTO). Es importante indicar que no sólo se evidenciaron incendios en más de una fecha, sino también que éstos estaban presentes en más de un sitio, como 1484 y 1893 en CTO y MAT; 1800 en CTO y URC (figura 4).

La cronología de $\mathrm{CCH}$ presentó una longitud de 754 años. Solamente una muestra alcanzó a cubrir el período hasta 1249 y recién en 1352 comenzaron las otras series a incluirse en la cronología (figura 5). El período mejor replicado se encontró entre 1509 y 1930, en donde el número de muestras varió entre 8 y 16. En los extremos se presentaron valores muy disímiles a la media (figura 5). Esto se vio relacionado con la cantidad notoriamente inferior de muestras observadas en la misma figura para los períodos antes mencionados. En URC la cronología del registro de anillos fue de 556 años y cubrió el período 1354-1910, pero sólo dos muestras de un total de nueve se extendieron hasta antes de 1495. El período mejor replicado en este

Cuadro 4. Orígenes y usos del fuego en el siglo XX, cuenca media del Cachapoal.

Origins and uses of fire in the twentieth century, Cachapoal River-middle basin.

\begin{tabular}{|c|c|c|c|}
\hline Fuente & Cita textual & Origen & Uso \\
\hline Ángel Lara, arriero & $\begin{array}{l}\text { "Eran los hijos de los dueños, estaban jugando... hicieron fuego } \\
\text { y se les quemó una mancha de cipreses en Urriola... y como } \\
\text { ellos eran cabros, no se midieron... ¡y se les fue no más!" }\end{array}$ & $\begin{array}{l}\text { Antrópico. } \\
\text { Accidental }\end{array}$ & - \\
\hline $\begin{array}{l}\text { Salomé Silva, dueña } \\
\text { de Casa }\end{array}$ & $\begin{array}{l}\text { "Los antiguos usaban hartazo el fuego para esos lados... eso } \\
\text { me contaba mi Papá... Quemaban harto." }\end{array}$ & $\begin{array}{l}\text { Antrópico. } \\
\text { Intencional }\end{array}$ & - \\
\hline $\begin{array}{l}\text { Joaquín López, } \\
\text { manutención CONAF }\end{array}$ & $\begin{array}{l}\text { "La otra vez vimos un fuego natural... había una tormenta y } \\
\text { caían los rayos y había harto pasto, así que empezó rapidito } \\
\text { un incendio..." }\end{array}$ & $\begin{array}{l}\text { Natural. } \\
\text { Tormentas }\end{array}$ & - \\
\hline Ramón López, arriero & $\begin{array}{l}\text { "Para allá hace frío también y lo usábamos para calentarse } \\
\text { y cocinar... para leña... a alguno se le pasaba la mano y se } \\
\text { quemaba de más..." }\end{array}$ & $\begin{array}{l}\text { Antrópico. } \\
\text { Accidental }\end{array}$ & $\begin{array}{l}\text { Fogatas (calefacción y } \\
\text { cocina) }\end{array}$ \\
\hline $\begin{array}{l}\text { Rosa Miranda, dueña } \\
\text { de casa }\end{array}$ & $\begin{array}{l}\text { "Y quemaban unas manchitas para hacerse señas... Adonde } \\
\text { andaba el viejo que estaba rodeando adentro y hacía un humo } \\
\text { para que el que estaba más abajo o en el otro lado supiera } \\
\text { donde estaba..." }\end{array}$ & $\begin{array}{l}\text { Antrópico. } \\
\text { Intencional }\end{array}$ & Ubicación de arrieros \\
\hline Ángel Lara, arriero & $\begin{array}{l}\text { "Cuando bajaban el ganado hacían fuego para quemar el } \\
\text { clonque iy se quemaban todas las cosas ahí poh!..." }\end{array}$ & $\begin{array}{l}\text { Antrópico. } \\
\text { Intencional }\end{array}$ & Despeje \\
\hline Ramón López, arriero & $\begin{array}{l}\text { "Los viejos quemaban para buscar los 'animales lobo', esos } \\
\text { que se metían debajo de los cipreses para esconderse... ¡Ahí } \\
\text { quemaban y salían arrancando los animales!..." }\end{array}$ & $\begin{array}{l}\text { Antrópico. } \\
\text { Intencional }\end{array}$ & Búsqueda de animales \\
\hline
\end{tabular}




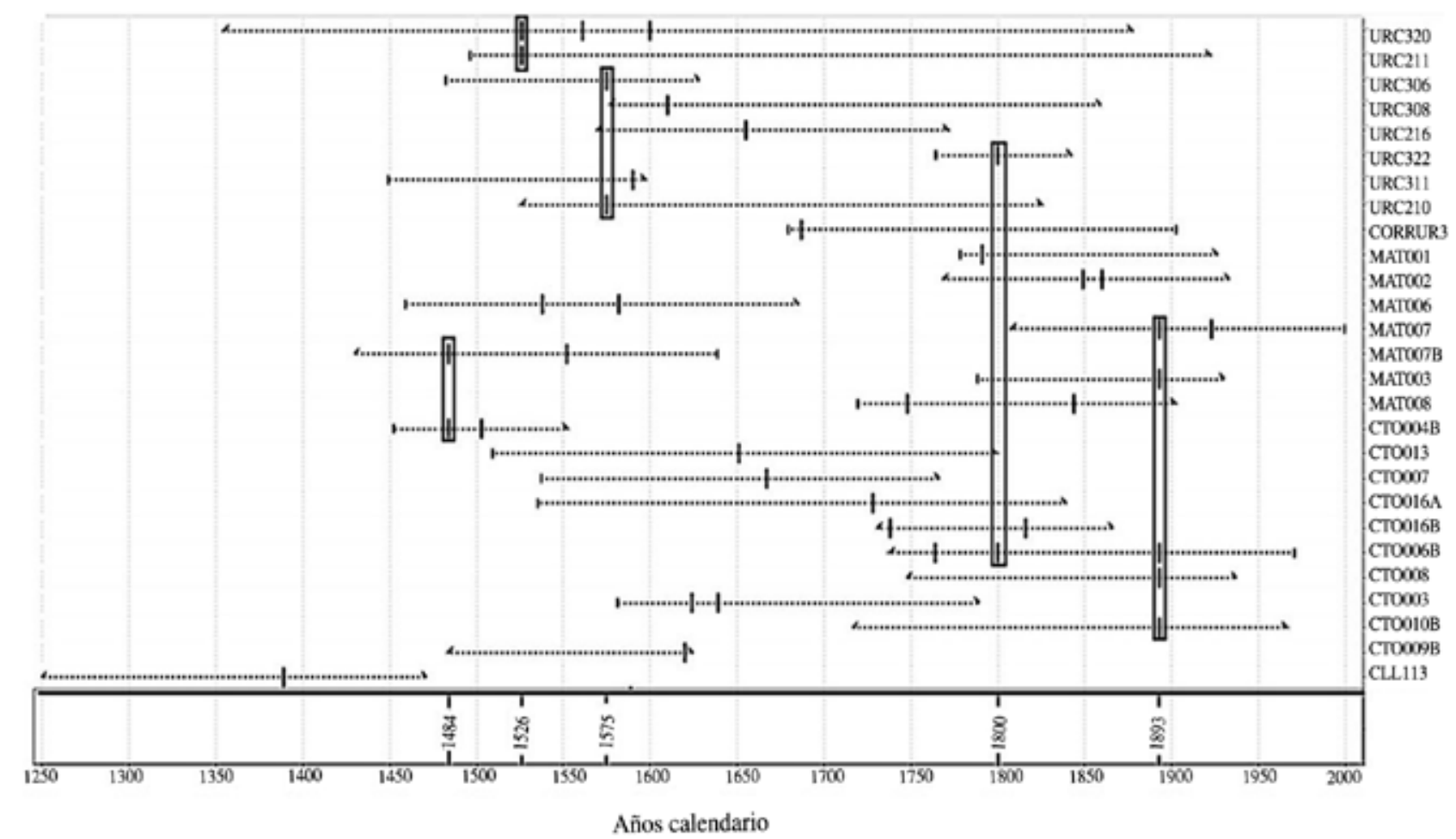

Figura 4. Muestras datadas y sus respectivas fechas de incendio para Austrocedrus chilensis en la cuenca media del Cachapoal. Cada línea horizontal representa una muestra datada, en donde los eventos de incendios se indican con una línea vertical. Los segmentos punteados de las líneas horizontales representan el crecimiento del árbol previo al primer fuego registrado, los de línea continua indican crecimiento del árbol post incendio. Las líneas diagonales al comienzo de cada línea horizontal representan la ausencia de médula en la muestra, la línea vertical, presencia de ésta. Las líneas diagonales a la derecha simbolizan ausencia de corteza, las verticales, presencia. Los años calendario listados e indicados con recuadro representan años con dos o más eventos de incendio. Gráfico realizado con el programa FHX2 (Grissino-Mayer 2001).

Dated Austrocedrus samples from the Cachapoal River middle basin. Each horizontal line represents a dated sample, with the fire events indicated by vertical short lines. The segments of the dotted lines represent the growth of the tree without the intervention of fire; the continuous line indicates tree growth after fire. The diagonal lines at the beginning of each horizontal line represent the absence of bone marrow in the sample; the vertical line, the presence of it. The diagonal lines on the right symbolize the absence of bark, vertical presence. Marks enclosed by vertical boxes represent years where fires are present in two or more samples. Graphic made with FHX2 program (Grissino-Mayer 2001).

sitio fue entre 1525 y 1856 (figura 5). En este período el número de muestras varió entre cuatro y nueve. Se observó que la estandarización para los años de fines del siglo XIX se tornó muy heterogénea y disímil, afectada claramente por la baja cantidad de muestras que representaban este período. La longitud del registro de anillos para MAT fue de 575 años e incluyó el período 1429-2003, pero existió una ausencia de muestras que abarcaban el período 16831718. Entre 1429 y 1682 existieron sólo dos muestras. En el tramo 1807-1918 se presentó la mayor cantidad de muestras, cinco rodelas. La cronología del sitio CTO fue de 516 años, abarcando el período de tiempo entre 1452-1967. Solamente una rodela abarcó el período comprendido entre 1452 y 1481 . Entre 1493 y 1508 existieron dos muestras, misma cantidad que registró el período más joven de la cronología, 1874-1966. En CLL no se logró realizar una cronología, ya que solamente una muestra se logró datar confiablemente $(r=0,39 ; P<0,05)$. Esta rodela presentó 223 años comprendidos entre 1249 y 1389 (figura 5). La muestra presentó un intervalo de reducción en índice de ancho de anillo entre 1320 y 1380, situación que se repitió desde 1414 a 1434.

En $\mathrm{CCH}$ existieron 39 cicatrices de incendio en los 754 años de longitud de la serie. Estas cicatrices comprendieron 34 años diferentes de eventos de incendio, por lo cual resulta que el intervalo medio de incendios fue de 22 años (cuadro 5). Como CLL correspondió sólo a una muestra, resultó un alto intervalo de retorno de fuego con 


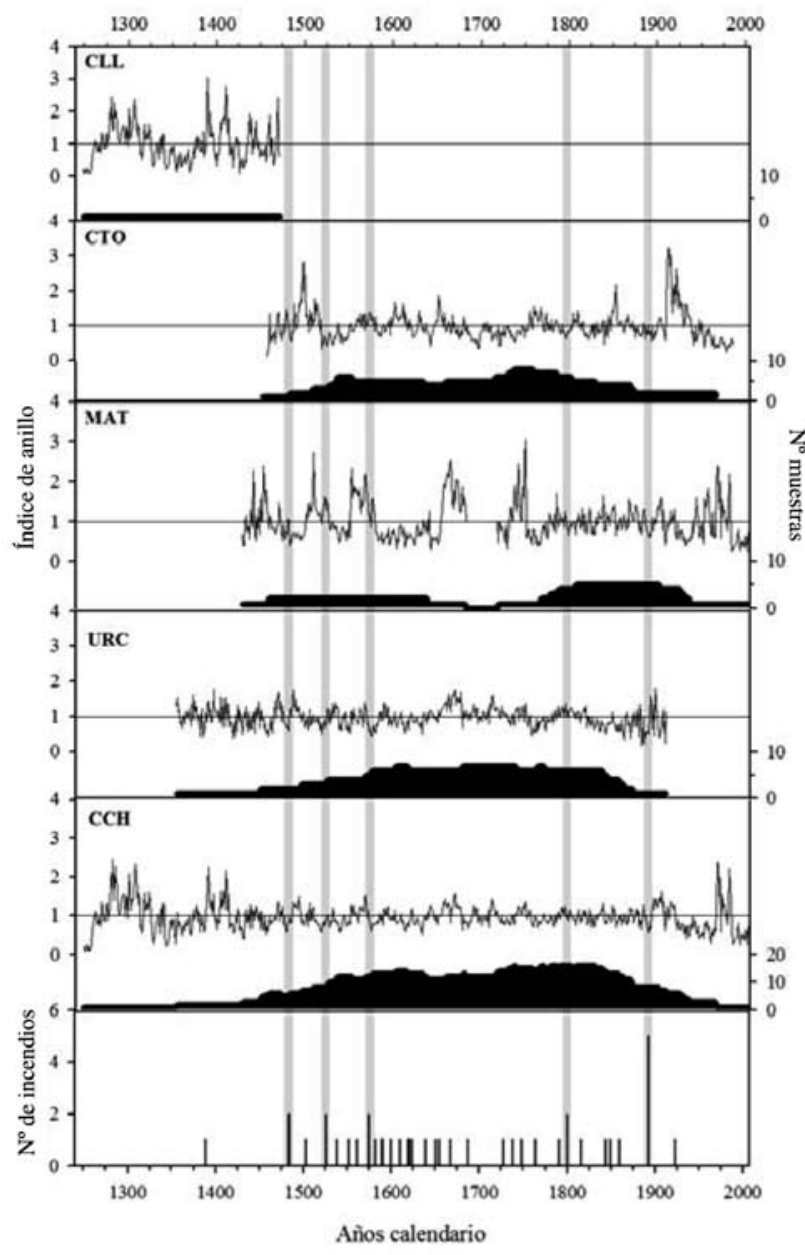

Figura 5. Cronologías de anchos de anillos de los cuatro sitios en la cuenca del río Cachapoal. Las cronologías poseen media 1 (línea horizontal) y fueron estandarizadas mediante el ajuste de las series a su crecimiento medio (estandarización horizontal). Se muestra a su vez el número de muestras por año para la cuenca y por sitio en el eje Y derecho. Bajo los gráficos de cronologías se muestra el número de cicatrices de incendios por año registrados en los cuatro sitios. Las zonas destacadas con gris representan la respuesta en el índice de anillo de los árboles en el año de incendio y el posterior a éste. Figura basada en Barichivich (2005).

Tree-ring-chronology widths of the four sites in the Cachapoal River middle basin. Timelines have averaged 1 (horizontal line) and were standardized by adjusting the series to their mean growth (horizontal standardization). Numbers of samples per year are indicated on the right vertical axis. The bottom graph shows the number of fire scars for year. The areas highlighted in gray represent the response of the tree-ring growth during the forest fire year and in the following few years. Figure based on Barichivich (2005).
140 años. MAT y CTO son los que presentaron la mayor cantidad de años de incendio, 11 y 13 respectivamente, y con ello las más bajas tasas de retorno de fuego, 52 años para MAT y 40 años para CTO. URC presentó 11 cicatrices, nueve años diferentes de incendios con una frecuencia de incendios de uno cada 62 años.

\section{DISCUSIÓN}

El fuego en esta cuenca es un proceso importante en el funcionamiento del ecosistema y un claro modelador del paisaje desde épocas prehispánicas, antes de la llegada de jesuitas y conquistadores. Los incendios sincrónicos encontrados en CTO y MAT, así como los de CTO y URC (figura 4) responderían probablemente a factores climáticos. Por ejemplo, 1893 se relaciona a un año inserto en un periodo seco, tanto para Chile central como para el norte de la Patagonia argentina (Villalba et al. 1998, Lara et al. 2000). Aravena et al. (2003) señalan, para el mismo año, incendios en bosques de Austrocedrus chilensis en Alto Biobío ( $\left.38^{\circ} \mathrm{S}, 71^{\circ} \mathrm{O}\right)$. Le Quesne et al. (2006) no sólo identifican a 1892 como uno de los 10 años más secos en Chile central, sino que mencionan que las cronologías derivadas de sitios de esta zona geográfica se encuentran altamente correlacionadas con las precipitaciones estacionales. Además de los datos paleoclimáticos que indican sequía pronunciada y los datos del Biobío de Aravena et al. (2003), en 1893 el $31 \%$ de los sitos del NO de la Patagonia argentina muestran fuego (Veblen et al. 1999). Por otra parte, 1893-94 están catalogados con base en reconstrucciones multiproxy recientes como de La Niña extrema (Gergis y Fowler 2006). Este tipo de factores climáticos generó condiciones de sequía extrema que habrían favorecido la propagación de los incendios que provocaron en 1484, 1800 y 1893 disturbios a escala regional. Esta idea podría reafirmarse a partir de la depresión en el crecimiento en URC, en donde no se verifican cicatrices de incendio para el año 1893 (figura 5).

La revisión histórica señala que en 1893 hubo un cambio cultural en la producción de las haciendas de esta cuenca, desde ganadería a agricultura mixta (cuadro 3), lo que pudo agregar mayor presión y favorecer la ocurrencia

Cuadro 5. Intervalo de incendios en la cuenca media del Cachapoal.

Fire frequency in the Cachapoal river middle basin.

\begin{tabular}{lcccccrr}
\hline Sitio & $\begin{array}{c}\text { Año } \\
\text { inicio }\end{array}$ & $\begin{array}{c}\text { Año } \\
\text { final }\end{array}$ & $\begin{array}{c}\text { Longitud } \\
\text { (años) }\end{array}$ & $\begin{array}{c}\mathrm{N}^{\circ} \\
\text { cicatrices }\end{array}$ & $\begin{array}{c}\text { Años por } \\
\text { cicatriz }\end{array}$ & $\begin{array}{c}\mathrm{N}^{\circ} \\
\text { incendios }\end{array}$ & $\begin{array}{c}\text { Intervalo de } \\
\text { incendios (años) }\end{array}$ \\
\hline URC & 1354 & 1910 & 556 & 11 & 51 & 9 & 62 \\
MAT & 1429 & 2003 & 574 & 12 & 48 & 11 & 52 \\
CTO & 1452 & 1967 & 515 & 15 & 34 & 13 & 1 \\
CLL & 1249 & 1389 & 140 & 1 & 140 & 140 \\
CCH & 1249 & 2003 & 754 & 39 & 19 & 34 & 22 \\
\hline
\end{tabular}


y extensión del fuego mediante el despeje de vegetación nativa. Otro factor que puede incidir en los incendios de MAT y CTO es que se encuentran a menor altitud y más cerca de los centros poblados (figura 1). Esto, a su vez, podría explicar la baja tasa de retorno de incendios, comparado con los otros dos sitios, tal como lo mencionan Montenegro et al. (2002) y Aravena et al. (2003).

Los incendios registrados en sólo un año pueden resultar de eventos locales, de poca extensión, ya que estos bosques están conformados por un mosaico de pequeños bosquetes de distribución dispersa (Le Quesne et al. 2000). A su vez, coinciden con las fluctuaciones del crecimiento radial presentadas en la cronología (figura 5), en donde el patrón de estas variaciones se asemeja al presentado por las cronologías de Le Quesne et al. (2000) para el sitio Río Clarillo y de Lara et al. (2001) para los sitios de Vilches y laguna del Laja. Esto quiere decir que la curva de la cronología de la cuenca es diferente a la media 1, ya sea con valores de índice superiores o inferiores. Dichas variaciones son más marcadas en MAT entre 1683 y 1718, asociadas además a la disminución del tamaño muestral. En los períodos en donde la cantidad de muestras de la cronología es estable (1509-1930; de ocho a 16 muestras), existe una coincidencia entre las fluctuaciones de crecimiento y la presencia de incendios (figura 5). Estos patrones de crecimiento radial estarían asociados al régimen de incendios en cada sitio, y luego de cada evento de fuego, los árboles responden con una abrupta liberación en el crecimiento, debido a una mayor disponibilidad de recursos por la muerte de árboles vecinos (Le Quesne et al. 2000). Otros factores que explicarían los eventos de incendio a escala local serían: i) baja densidad y dispersión de los individuos (Le Quesne et al. 2000); ii) escasa acumulación de combustible bajo los bosquetes de Austrocedrus chilensis (Bustos 2007); iii) variación de las temperaturas entre invierno y verano (Villalba y Veblen 1997), que favorece el termoclastismo que genera gran cantidad de sedimento granítico y piroclástico grueso (Bustos 2007); iv) las especiales características de la flora acompañante de estos bosques de Austrocedrus chilensis, que presentan una mayor cantidad de plántulas de especies arbóreas que brinzales de Austrocedrus chilensis y escasa presencia de individuos de esta especie en etapa fustal (Barría 2007).

Después de 1923 (figura 4) no se observan evidencias de fuego en la cronología, lo que no significa que no hayan existido, sino que el número de muestras que cubre dicho período es muy bajo en el presente estudio (3). El registro de los mismos puede existir en individuos vivos de Austrocedrus chilensis de los sitios estudiados. Por ello, sería de gran importancia en futuras investigaciones incluir tarugos de árboles vivos y más rodelas de individuos muertos con o sin evidencias de incendios en la parte exterior del fuste. Además, sería de gran utilidad la inclusión de otros rodales de Austrocedrus chilensis presentes en el área y que no fueron muestreados en esta oportunidad, para realizar una cronología más robusta de esta cuenca. Esto favorecería tanto a la extensión de esta cronología como la datación de eventos de incendio más recientes, que permitan comprender mejor la historia de este disturbio en los sitios analizados.

\section{CONCLUSIONES}

El fuego en esta zona es parte importante del ecosistema y claro modelador del paisaje, por ser un elemento que habría estado presente durante todo el Holoceno, desde el período inicial de cazador- recolector hasta la actualidad.

Las entrevistas señalan que el fuego ha sido un elemento ampliamente usado por la población rural, especialmente para fines de calefacción y preparación de alimentos (fogatas), ubicación (señales de humo) y despeje para la búsqueda de animales. Esto indica una continua utilización de la cubierta vegetal en la región.

Existen evidencias que permiten relacionar estrechamente las actividades antropogénicas y las condiciones climáticas con la ocurrencia y frecuencia de incendios en la cuenca media del río Cachapoal. Esta investigación constituye la primera base para desarrollar una cronología de incendios en el área de estudio y, a pesar de su carácter exploratorio, muestra el gran potencial que posee este extraordinario archivo natural -contenido en las cicatrices de la madera de ciprés- para contribuir a la historia natural y cultural de Chile central.

\section{AGRADECIMIENTOS}

Al proyecto FONDECYT 3020011 y al Proyecto International Foundation for Science (IFS, D/3124-2). A Pamela Ramos Valdés por su valiosa ayuda y compañía en la formulación de este escrito. A Violeta, mi hija, por su compañía. A Don Joaquín López, Julio César Vergara y a todos los guardaparques de la RNRC.

\section{REFERENCIAS}

Arrué L. 2000. Historia del territorio que hoy ocupa la Reserva Nacional Río de Los Cipreses. Rancagua, Chile. CONAF (Corporación Nacional Forestal, CL). 84 p.

Aravena JC, C Le Quesne, H Jiménez, A Lara, J Armesto. 2003. Fire history in Central Chile: Tree ring evidence and modern records. In Veblen T, W Baker, G Montenegro, $\mathrm{T}$ Swetnam eds. Fire and Climatic Change in Temperate Ecosystems of the Western Americas. Cambridge, EEUU. Springer. p. 343-356.

Barichivich J. 2005. Muerte apical episódica en bosques de alerce (Fitzroya cupressoides [Mol.] Johnston) de la Cordillera de la Costa de Valdivia. Tesis Ingeniero Forestal. Valdivia, Chile. Facultad de Ciencias Forestales, Universidad Austral de Chile. 54 p. 
Barría J. 2007. Estructura y regeneración de Austrocedrus chileLnsis (D. Don) Pic. Serm Bizzarri (Ciprés de la Cordillera), en los Corrales de Urriola, Reserva Nacional Río de Los Cipreses ( $\left.34^{\circ} 43^{\prime} \mathrm{S}\right)$. Tesis Ingeniero Forestal. Valdivia, Chile. Facultad de Ciencias Forestales, Universidad Austral de Chile. 38 p.

Bustos C. 2007. Historia de incendios en bosques de Austrocedrus chilensis (D Don) Pic. Ser. et Bizz. en la cuenca andina del río Cachapoal, Chile. Tesis Ingeniero Forestal. Valdivia, Chile. Facultad de Ciencias Forestales, Universidad Austral de Chile. 43 p.

Domeyko I. 1849. Viajes a las Cordilleras de Talca y de Chillán. Santiago, Chile. Fac. de Medicina y de Ciencias Físicas y Matemáticas, Universidad de Chile. $2^{\text {a }}$ Parte. 28 p.

Durán E, M Planella. 2000. Consolidación agroalfarera: Zona central. In Hidalgo J, V Schiappacasse, $\mathrm{H}$ Niemeyer, C Aldunate, I Solimano eds. Culturas de Chile. Prehistoria: Desde sus orígenes hasta los albores de la conquista. Santiago, Chile. Andrés Bello. p. 313-327.

Echevarría E. 1988. Leyendas de los Andes de Chile. Santiago, Chile. Arancibia. 155 p.

Falabella F. 1994. La Cordillera de los Andes: Ruta de Encuentros. Dos puntas tiene el camino: Antiguas relaciones trasandinas en el centro de Chile y Argentina. Santiago, Chile. Museo Chileno de Arte Precolombino. 96 p.

Falabella F, R Stehberg. 2000. Los inicios del desarrollo agrícola y alfarero: Zona central. In Hidalgo J, V Schiappacasse, H Niemeyer, C Aldunate, I Solimano eds. Culturas de Chile. Prehistoria: Desde sus orígenes hasta los albores de la conquista. Santiago, Chile. Andrés Bello. p. 295-311.

Fritts H. 1976. Tree-ring and climate. Arizona, EEUU. Laboratory of Tree-Ring Research. University of Arizona. 567 p.

Fuenzalida A. 1919. El trabajo i la vida en el mineral El Teniente. Santiago, Chile. Sociedad. Imprenta-Litografía Barcelona. $211 \mathrm{p}$.

Gay C. 1980. Imágenes de Chile: 10 láminas del Atlas de la Historia física y política de Chile. Santiago, Chile. Universitaria. $7 \mathrm{p}$.

Gergis JL, AM Fowler. 2006. How unusual was late 20th century El Niño-Southern Oscillation (ENSO)? Assessing evidence from tree-ring, coral, ice-core and documentary palaeoarchives, A.D. 1525-2002. Advances in Geosciences 6: $173-179$.

Grissino-Mayer H. 2001. FHX2: Software for analyzing temporal and spatial patterns in fire regimes from tree rings. Treering Research 57(1): 115-124.

Grissino-Mayer H. 2006. Ultimate tree-rings pages: Principles of Dendrochronology. Definitions. Consultado 28 feb. 2007. Disponible en http://web.utk.edu/ grissino/principles.htm.

Grissino-Mayer H, H Blount, A Miller. 2001. Tree-ring dating and the ethnohistory of the naval stores industry in southern Georgia. Tree Ring Research 57(1): 3-13.

Guyette R, D Dey. 1995. A presettlement fire history in an Oak-Pine forest near Basin Lake, Algonquin Park, Ontario. Forest Research Report 132, Canada. 7 p.

Hanisch W. 1974. Historia de la Compañía de Jesús en Chile. Santiago, Chile. Francisco de Aguirre. 263 p.

Holmes R, R Adams, H Fritts. 1986. Quality control of crossdating and measuring: a users manual for the program COFECHA. In Tree-ring chronologies of western North America: California, eastern Oregon and northern Great
Basin. Arizona, EEUU. Laboratory of Tree-Ring Research, University of Arizona. $9 \mathrm{p}$

Johnson F, C Van Wagner. 1978. The theory and use of two fire history models. Can. J. For. Res. 15: 214-220.

Kitzberger T, TT Veblen, R Villalba. 1997. Climatic influences on fire-regimes along a rain forest-to-xeric woodland gradient in northern Patagonia, Argentina. J. of Biogeography 24(1): 35-47.

Lachmund H. 1921. Some phases in the formation of fire scars. J. of Forestry 19(6): 638-640.

Lara A, JC Aravena, R Villalba, A Wolodarsky-Franke, B Luckman, R Wilson. 2001. Dendroclimatology of highelevation Nothofagus pumilio forests and their northern distribution limit in the central Andes of Chile. Can. J. For. Res. 31: 925-936.

Lara A, R Villalba, JC Aravena, A Wolodarsky-Franke, E Neira. 2000. Desarrollo de una red de Cronologías para Fitzroya cupressoides (Alerce) para Chile y Argentina. In: Roig F ed. Dendrocronología en América Latina. Mendoza, Argentina. EDIUNC. p. 217-244.

Larraín de Castro C. 1982. La familia Larraín: sus orígenes en España e historial de la rama mayor de Chile. Santiago, Chile. Universitaria. 339 p.

Latcham R. 1928. La prehistoria chilena. Santiago, Chile. Universo. 238 p.

Le Quesne C. 1988. Caracterización de bosques de ciprés de la cordillera (Austrocedrus chilensis (D. Don) Florin et Boutelje), en Radal-7 Tazas, Séptima Región, Chile. Tesis Ingeniero Forestal. Valdivia, Chile. Facultad de Ciencias Forestales, Universidad Austral de Chile. 66 p.

Le Quesne C, JC Aravena, M Álvarez, J Fernández. 2000. Dendrocronología de Austrocedrus chilensis (Cupressaceae) en Chile central. In Roig F ed. Dendrocronología en América Latina. Mendoza, Argentina. EDIUNC. p. 159-175.

Le Quesne C, JC Aravena, H Jiménez, R Villa, JC Vergara. 1999. Caracterización de un rodal de Austrocedrus chilensis (D. Don) Pic. Ser. et Bizz. en la Reserva Nacional Río de Los Cipreses, VI Región, Chile. (Serie Técnica No 3(2)). 19 p.

Le Quesne C, D Sthale, M Cleaveland, M Therrell, JC Aravena, J Barichivic. 2006. Ancient Austrocedrus Tree-Ring Chronologies Used to Reconstruct Central Chile Precipitation Variability from A.D. 1200 to 2000. J. of Climate 19(22): 5731-5744.

Medina JT. 1952. Los aborígenes de Chile. Santiago, Chile. Universitaria. $431 \mathrm{p}$.

Montenegro G, F Díaz, M Gómez, R Ginocchio. 2002. Regeneration potential of Chilean matorral after fire: an updated view. In Veblen T, W Baker, G Montenegro, T Swetnam eds. Fire and climate change in temperate ecosystems of the western Americas. New York, EEUU. Springer-Verlag. p. 375-403.

Montenegro G, M Gómez, R Ginocchio, A Segura, J Keely. 2007. Regímenes de incendios y respuestas de la vegetación en dos regiones de clima mediterráneo. Documento Técnico $\mathrm{N}^{\mathrm{o}}$ 179. Santiago, Chile, CONAF (Corporación Nacional Forestal, CL). 12 p.

Niemeyer H, P Cereceda. 1984. Geografía de Chile: Hidrografía. Tomo VIII. Santiago, Chile. Instituto Geográfico Militar. $320 \mathrm{p}$.

Novoa R, S Villaseca. 1989. Mapa Agroclimático de Chile. Santiago, Chile. Instituto de Investigaciones Agropecuarias (INIA, CL). 221 p. 
Núñez L, J Varela, R Casamiquela, V Schiapacasse, H Niemeyer, C Villagrán. 1994. Cuenca de Tagua Tagua en Chile: el ambiente del Pleistoceno y ocupaciones humanas. Revista Chilena de Historia Natural 67(4): 117-125.

Philippi RA. 1875. Historia Natural: Excursión al cajón de Los Cipreses en la hacienda de Cauquenes (Rancagua). Anales de la Universidad, Memorias científicas y literarias. Santiago, Chile. Universidad de Chile. 26 p.

Pissis A. 1860. Descripción topográfica i jeográfica de la provincia de Colchagua. Santiago, Chile. Imprenta Nacional. 45 p.

Stokes M, T Smiley. 1968. Tree-ring dating. Chicago, EEUU. University of Chicago Press, $73 \mathrm{p}$.

Taylor S, R Bogdan. 1987. Introducción a los métodos cualitativos de investigación. Barcelona, España. Paidos Ibérica. $343 \mathrm{p}$.

Veblen TT, T Kitzberger. 2002. Inter-hemispheric comparison of fire history: The Colorado Front Range, U.S.A., and the Northern Patagonian Andes, Argentina. Plant Ecology 163: $187-207$.
Veblen TT, T Kitzberger, R Villalba. 2004. Nuevos paradigmas en ecología y su influencia sobre el conocimiento de la dinámica de los bosques del sur de Argentina y Chile. In Arturi MF, JL Frangi, JF Goya eds. Ecología y Manejo de los Bosques de Argentina. La Plata, Argentina, EDULP. Presentación multimedia.

Veblen TT, T Kitzberger, R Villaba, J Donnegan. 1999. Fire history in northern Patagonia: The roles of humans and climatic variation. Ecological Monographs 69(1): 47-67.

Villalba R, E Cook, G Jacoby, R D’Arrigo, TT Veblen, Jones. 1998. Tree-ring based reconstructions of northern Patagonia precipitation since AD 1600. The Holocene 8(6): 659-674.

Villalba R, T Veblen. 1997. Regional patterns of tree population age structure in northern Patagonia: Climatic and disturbance influences. J. of Ecology 85(2): 113-124.

Yrarrázabal J. 1940. El Marqués de Larraín y su descendencia. Santiago, Chile. Imprenta Chile. 110 p.

Recibido: 23.06 .09

Aceptado: 16.10 .09 